AperTO - Archivio Istituzionale Open Access dell'Università di Torino

\title{
Antilisterial Effect and Influence on Listeria monocytogenes Gene Expression of Enterocin or Enterococcus faecalis in Sliced Dry-Cured Ham Stored at 78C
}

\section{This is the author's manuscript}

Original Citation:

Availability:

This version is available http://hdl.handle.net/2318/1734693

since 2020-04-02T10:40:35Z

Published version:

DOI:10.4315/0362-028X.JFP-19-024

Terms of use:

Open Access

Anyone can freely access the full text of works made available as "Open Access". Works made available under a Creative Commons license can be used according to the terms and conditions of said license. Use of all other works requires consent of the right holder (author or publisher) if not exempted from copyright protection by the applicable law. 
1 Running title: Antilisterial effect of enterocin in sliced dry cured ham

2

3 Antilisterial effect and influence on Listeria monocytogenes gene expression of 4 enterocin or Enterococcus faecalis in sliced dry cured ham stored at $7{ }^{\circ} \mathrm{C}$

5

6 Raquel Montiel1§, Ana Quesille-Villalobos²§, Valentina Alessandria ${ }^{3}$, Margarita Medina ${ }^{1}$, Luca

$7 \quad$ Simone Cocolin ${ }^{3}$, Kalliopi Rantsiou ${ }^{3 *}$

8

9

101 Department of Food Technology, National Institute for Agricultural and Food Research and

11 Technology (INIA), Spain

$122^{2}$ Laboratory of Microbiology and Probiotics, Institute of Nutrition and Food Technology

13 (INTA), University of Chile, Chile

$143^{3}$ Department of Agricultural, Forest and Food Sciences, University of Torino, Italy

15

16

17 Keywords: biopreservation, enterocin, Listeria monocytogenes, gene expression, dry-cured 18 ham

21 §authors contributed equally to the work

23 *Corresponding author: kalliopi.rantsiou@unito.it, Largo Paolo Braccini 2, 10095 Grugliasco,

24 Italy, 0039-011-6708870 
In this study we focused on the effect of an enterocin or an Enterococcus faecalis strain added onto sliced dry cured ham that was artificially inoculated with L. monocytogenes and stored at $7{ }^{\circ} \mathrm{C}$. The population of L. monocytogenes and the expression of 5 genes were monitored throughout the storage period. A persistent and a non-persistent strain were tested and both were influenced by the presence of the enterocin; both populations were reduced by more than $2 \log _{10} \mathrm{CFU} / \mathrm{g}$ after 14 days, compared to the control, non-inoculated ham. The presence of E. faecalis, a bacteriocin producing lactic acid bacterium, had a much less pronounced effect on the viable counts for both strains. Concerning gene expression, a common trend that was observed for both strains in the presence of enterocin was the downregulation of genes tested after 30 minutes of storage at $7{ }^{\circ} \mathrm{C}$. For the remaining of the storage period the expression fluctuated but was mostly reduced. Similarly, the presence of E. faecalis led to an overall downregulation of genes. The effect on gene expression of both the enterocin and the $E$. faecalis was more pronounced on the non-persistent L. monocytogenes strain. Although the potential of a bacteriocin and a bacteriocin producing microorganism to control $L$. monocytogenes was confirmed, this study highlights that gene expression may be influenced and needs to be evaluated when considering such biopreservation interventions.

Keywords: biopreservation, enterocin, Listeria monocytogenes, gene expression, dry-cured ham 
51

52 


\section{Highlights}

$54 \quad$ - Viability of Listeria monocytogenes in sliced dry-cured ham was greatly influenced by the addition of an enterocin

- Addition of a bacteriocin producing Enterococcus faecalis had a less pronounced effect on L. monocytogenes viability

- Expression of genes related to L. monocytogenes stress response/adaptation was modified in the presence of an enterocin

60

- The addition of a bacteriocin producing Enterococcus faecalis influenced gene expression in one of the two L. monocytogenes strains tested 
63 The term biopreservation or biological preservation of foods was coined in the mid-90's and refers to the food safety improvement and extension of shelf life through microbial antagonism $(27,28)$. A strong antagonistic ability is attributed to lactic acid bacteria (LAB) and has been documented for a variety of fermented foods (16). Inhibition of undesirable microorganisms can be due to direct effect of LAB through competition for nutrients, niche occupation or indirect effect through synthesis of bacteriocins and/or production of other metabolites. More than 20 years of research have expanded our knowledge regarding the modes of action of LAB naturally present in the foods or intentionally added as protective cultures. Further, the field of application of LAB and/or associated bacteriocins has been broadened to include non-fermented foods, food plant environment but also employment in non-food sectors (4).

Many bacteriocins produced by LAB exert an inhibitory action towards strains of Listeria monocytogenes, a foodborne pathogen of particular concern for refrigerated ready-to-eat (RTE) foods. For this reason, LAB bacteriocins with antilisterial effect have been the focus of both in vitro and in situ studies to understand the potential for industrial application to reduce the L. monocytogenes risk associated with RTE foods. Efficacy of bacteriocins, or overall LAB competition, in inhibiting or reducing L. monocytogenes growth in various RTE foods is well documented and is reviewed by Zilelidou and Skandamis (35). However, most of the studies so far conducted examined how bacteriocins or LAB impact on growth parameters of L. monocytogenes not taking into consideration the consequences for the physiology of the microorganism. Therefore there is the need to integrate current knowledge regarding the antilisterial effect with information concerning molecular/cellular response of $L$. monocytogenes to LAB and/or bacteriocin presence or addition in foods. A potential first step in appreciating changes in microbial physiology is by looking into changes in gene expression (9). 
The purpose of this study was dual. First, we compared the antilisterial effect of an enterocin and an E. faecalis strain, added to sliced dry-cured ham and incubated at refrigeration temperature. Secondly, we evaluated the expression of genes that are involved in stress response and adaptation, under the same conditions. Two different strains of $L$. monocytogenes isolated from a meat plant environment were tested; one was previously shown to be persistent and the other one non-persistent (23).

\section{Materials and methods}

\section{Bacterial strains and culture media}

Two Listeria monocytogenes strains, previously isolated from an Iberian pig processing plant, were used in this study and belonged to the culture collection of INIA (Instituto Nacional de Investigación y Tecnología Agraria y Alimentaria, Madrid, Spain). Strain S4-2 was serotype $1 / 2 \mathrm{~b}$ and has been characterized as persistent in the environment while strain S12-1 was serotype $1 / 2 \mathrm{c}$ and non-persistent (23). The strains were maintained as stock cultures at -80 ${ }^{\circ} \mathrm{C}$ in Trypticase Soy Yeast Extract Broth (TSYEB, Biolife s.r.l., Milano, Italy) supplemented with $15 \%$ glycerol. Before use in experiments, strains were sub-cultured twice onto Brain Heart Infusion agar (BHI, LabM Ltd., Lancanshire, UK) at $37{ }^{\circ} \mathrm{C}$ for 24 hours. A bacteriocinogenic strain of Enterococcus faecalis was also used. This strain, E. faecalis B1, was previously isolated from raw bovine meat, identified to the species level by sequencing of the gene encoding the 16S rRNA and belonged to the culture collection of the University of Turin, Italy. The E. faecalis strain was maintained as a stock culture at $-80{ }^{\circ} \mathrm{C}$ in $\mathrm{M} 17$ Broth (Oxoid, Milan, Italy), supplemented with 15\% glycerol. Before use in experiments, the strain was subcultured twice onto M17 agar at at $37{ }^{\circ} \mathrm{C}$ for 24 hours. In addition, an enterocin extract was used in the experiments. The enterocin $\mathrm{AB}$ extract was previously obtained from an overnight 
113 culture of Enterococus faecium INIA TAB7 (26) at $30^{\circ} \mathrm{C}$ and semi-purified through ammonium 114 sulfate precipitation (300 g/L) (8) and stored at $-80{ }^{\circ} \mathrm{C}$ until use. The activity of the 115 bacteriocin extract was determined against the two L. monocytogenes strains through the agar 116 spot test (2) and expressed in arbitrary units (AU) per ml.

\section{Dry-cured ham preparation and inoculation}

119 One large piece ( $\sim 7 \mathrm{~kg})$ of dry-cured ham was purchased from a commercial supplier in Spain 120 and aseptically sliced in the laboratory. A sample was analyzed for the presence of $L$. 121 monocytogenes and resulted negative (absence in $25 \mathrm{~g}$ ). Subsequently, samples of $10 \mathrm{~g}$ of dry122 cured ham were inoculated by adding a cell suspension in Ringer's solution (Oxoid, Milan, 123 Italy) of L. monocytogenes S4-2 or S12-1 to achieve a final concentration of ca. $10^{6} \mathrm{cfu} / \mathrm{g}$. Cell 124 suspensions were prepared from overnight cultures in BHI. In a set of samples, the enterocin extract was added on the surface of the sliced dry-cured ham to reach a final activity of 1054 $\mathrm{AU} / \mathrm{g}$. For a second set of samples, a cell suspension of $E$. faecalis was added to reach a final concentration of ca. $10^{6} \mathrm{cfu} / \mathrm{g}$. Sliced dry-cured ham, inoculated with either of the two $L$. monocytogenes strains but not supplemented with enterocin or E. faecalis was used as control.

129 Samples were vacuum packed and maintained at $7{ }^{\circ} \mathrm{C}$ for 28 days. This temperature was 130 chosen taking into account literature data that suggest a higher than $4{ }^{\circ} \mathrm{C}$ temperature for 131 domestic refrigerators (12). Two biological replicates were considered for each strain of $L$. 132 monocytogenes, in each condition (i.e. enterocin or E. faecalis addition). By visual inspection, 133 no color differences were observed between the control and the enterocin or E. faecalis 134 supplemented ham during storage. Colour parameters $\left(\mathrm{L}^{*}, \mathrm{a}^{*}\right.$ and $\left.\mathrm{b}^{*}\right)$ in sliced dry-cured ham 135 with enterocin were previously studied and no significant changes were detected (22). 136 Average $\mathrm{pH}$ and $\mathrm{a}_{\mathrm{w}}$ values for this type of ham (as determined in previous experiments) are 5.9 and 0.905 , respectively. 


\section{Sampling during storage}

140 At time zero (immediately after inoculation) as well as after 6 hours, 7, 14 and 28 days of 141 storage at $7{ }^{\circ} \mathrm{C}$, a $10 \mathrm{~g}$ sample was subjected to microbiological analysis to determine the 142 viable count of L. monocytogenes. Briefly, the sample was transferred to a sterile stomacher 143 bag and $90 \mathrm{ml}$ of Ringer's solution were added. Then the sample was homogenized in a 144 stomacher (BagMixer, Interscience, France) for 2 minutes at normal speed and room 145 temperature. Serial decimal dilutions were prepared in the same solution and plated on 146 Listeria Selective Oxford Agar Base (Oxoid). Plates were incubated at $37{ }^{\circ} \mathrm{C}$ for 48 hours 147 before colony count. At time zero as well as after 30 minutes, 6, 24 and 168 hours (7 days) of 148 storage at $7^{\circ} \mathrm{C}, 10 \mathrm{~g}$ samples were used for RNA extraction and for the Agar Well Diffusion 149 Assay (AWDA) as described by Urso et al. (31). A homogenate was prepared, as described 150 above, from each $10 \mathrm{~g}$ sample. Two $\mathrm{ml}$ from the homogenate were centrifuged at 13,000 $\mathrm{g}$ for 1511 minute at $4{ }^{\circ} \mathrm{C}$. Immediately after centrifugation, the pellet was covered with $0.05 \mathrm{ml}$ of 152 RNAlater (Ambion, Applied Biosystems, Milan Italy) and stored at $-20{ }^{\circ} \mathrm{C}$ until the RNA 153 extraction.

\section{RNA extraction and cDNA synthesis}

156 RNA extraction was performed on the thawed samples, employing the procedure described 157 by Rantsiou et al. (24). Fifty microliters of lysozyme $(50 \mathrm{mg} / \mathrm{ml}$, Sigma) and $25 \mu \mathrm{l}$ of 158 proteinase $\mathrm{K}(25 \mathrm{mg} / \mathrm{ml}$, Sigma) were added to the thawed samples that were then incubated 159 at $37^{\circ} \mathrm{C}$ for 20 minutes in a Thermomixer compact (Eppendorf, Milan, Italy). Samples were 160 then processed using the MasterPure Complete DNA and RNA Purification Kit (Epicentre, 161 Madison, WI, USA), following the manufacturer's instructions. DNA was digested with the 162 Turbo DNase (Ambion) and complete removal of the DNA was verified by using an aliquot of 
the extract as template in a qPCR reaction (as described below). When amplification took 164 place, the DNase treatment was repeated until complete removal of the DNA. RNA was quantified using a Nanodrop ND-1000 spectrophotometer (Celbio, Milan, Italy). 166 Complementary DNA (cDNA) synthesis was performed using random hexamers (Promega, 167 Milan, Italy) according to Rantsiou et al. (24). The same quantity of RNA (ng/ $\mu$ l) was added in 168 the reaction for each sample. The M-MLV Reverse Transcriptase (Promega) was used 169 following the instructions of the manufacturer. An RNase Inhibitor (Promega) was added in 170 the reaction and dNTPs were added at a final concentration of $2 \mathrm{mM}$ each. Reverse 171 transcription was performed in a DNA Engine Peltier Thermal Cycler (BioRad, Milan, Italy) at $17237^{\circ} \mathrm{C}$ for 1 hour. The cDNA was stored at $-20^{\circ} \mathrm{C}$ until it was used in qPCR amplification.

\section{Quantitative PCR}

175 Quantitative PCR amplification was performed using the cDNA, synthesized as above from 176 each sample, as template. Five genes listed in Table 1 were targeted. The amplification took 177 place in a PCR Chromo4 Real Time PCR detection system (BioRad) using the SsoAdvanced SYBR Green Supermix (BioRad) and the amplification conditions described by Mataragas et al. (19) with the exception of the tuf gene annealing temperature that was adjusted to $55^{\circ} \mathrm{C}$. Each cDNA was amplified in triplicate, in the same amplification run, to reduce inter-run experimental variability.

\section{Data analysis - Statistical analysis}

184 Threshold cycle $\left(\mathrm{C}_{\mathrm{T}}\right)$ values were exported to Excel for further analysis. Mean $\mathrm{C}_{\mathrm{T}}$ values, for 185 each cDNA sample, were computed and used to calculate the relative gene expression by the 2 $186-\Delta \Delta \mathrm{C}_{\mathrm{T}}$ method, where $\Delta \Delta \mathrm{C}_{\mathrm{T}}$ is: $\left(\mathrm{C}_{\mathrm{T}} \text {, target }-\mathrm{C}_{\mathrm{T}} \text {, housekeeping }\right)_{\text {test condition }}-\left(\mathrm{C}_{\mathrm{T}} \text {, target }-\mathrm{C}_{\mathrm{T}} \text {, housekeeping }\right)_{\text {control }}$ 
housekeeping. Control condition was the sliced dry-cured ham inoculated with $L$. monocytogenes alone while the test condition was the dry-cured ham inoculated with $L$. monocytogenes and supplemented with enterocin or co-inoculated with E. faecalis (at the 191 respective time points). Log2 values of relative expression were calculated and statistically treated using the SPSS statistics (IBM Corp., Armonk, NY, USA).

\section{Results and Discussion}

195 Dry-cured ham is considered a ready to eat (RTE) food and it is known to be prone to Listeria 196 monocytogenes contamination during processing. The main hurdles to L. monocytogenes 197 growth during refrigerated storage are low $\mathrm{a}_{\mathrm{w}}$, addition of salt and nitrites. These hurdles 198 however are not listeriocidal and several studies have evaluated alternative approaches, with 199 a lethal effect, such as high hydrostatic pressure processing, irradiation and supercritical 200 carbon dioxide processing $(3,6,11,21)$. Furthermore, the potential of L. monocytogenes 201 growth control using bacteriocins has been investigated (13). In this study we sought to 202 investigate the behavior of L. monocytogenes in dry-cured ham supplemented with a 203 bacteriocin extract or co-inoculated with a bacteriocinogenic E. faecalis, during storage. In this 204 context, behavior is intended as population kinetics and gene expression profile during 205 storage. For this purpose, two strains of L. monocytogenes were tested; a persistent and a non206 persistent. The classification of the strains as persistent and non-persistent was based on 207 previous observations regarding frequency of isolation and occurrence in different areas of a 208 pig processing environment. More specifically, S4-2 was considered as a persistent strain 209 found in the environment, equipment, carcasses and raw and dry cured products. This genotype was repeatedly isolated. Strain S12-1 was non-persistent but isolated from dry

211 cured products (23). 
1. Effect of enterocin and bacteriocinogenic Enterococcus faecalis on Listeria monocytogenes population

By Agar Well Diffusion Assay performed in vitro it was determined that the enterocin extract and the bacteriocinogenic E. faecalis evenly inhibited both strains of L. monocytogenes (data not shown). When L. monocytogenes strains were artificially inoculated in dry-cured ham and stored under vacuum at $7{ }^{\circ} \mathrm{C}$, the viable count remained un-altered during the first 7 days and declined by about $0.6 \log _{10} \mathrm{CFU} / \mathrm{g}$ at 14 days (Table 2). The population then remained stable for both strains up to 28 days (data not shown). It has to be noted that previous works have determined that both $\mathrm{a}_{\mathrm{w}}$ and $\mathrm{pH}$ remain essentially unaltered during refrigerated storage of dry-cured ham. The average value of $\mathrm{pH}$ for the dry-cured ham was 5.9 while the average $\mathrm{a}_{\mathrm{w}}$ was 0.905. Further, salt and nitrites were added and during storage had average concentrations of $2.69 \mathrm{mg} / \mathrm{Kg}$ and $4.12 \%$ respectively. Taken together, these physicochemical characteristics render the product a food unable to support the growth of L. monocytogenes. Therefore, it is expected that a L. monocytogenes population, naturally present or artificially inoculated in such dry cured ham will remain stable or possibly decline with time during storage. Conversely, when the dry-cured ham was supplemented with enterocin, an immediate effect was observed in the population of L. monocytogenes. The population was reduced by almost $0.8 \log _{10} \mathrm{CFU} / \mathrm{g}$ for strain $\mathrm{S} 4-2$ and by $1.5 \log _{10} \mathrm{CFU} / \mathrm{g}$ for strain $\mathrm{S} 12-1$. It should be noted here that a time window of at least 30 minutes elapsed between the inoculation/enterocin supplementation and the sampling for the determination of the viable count. This time window was sufficient to observe the inhibition of L. monocytogenes. $L$. monocytogenes populations further declined at 7 and 14 days; the microbial load was reduced by $1.8 \log _{10} \mathrm{CFU} / \mathrm{g}$ between time 0 and 14 days for strain S4-2 and by $1.9 \log _{10} \mathrm{CFU} / \mathrm{g}$ for strain S12-1. At 14 days, the population of strain S4-2 was almost $2 \log _{10} \mathrm{CFU} / \mathrm{g}$ lower in the dry cured ham supplemented with enterocin compared to the control while for strain S12-1 
the effect was greater; the enterocin inactivated $2.8 \log _{10} \mathrm{CFU} / \mathrm{g}$ of the population. Therefore, the enterocin displayed significant listericidal effect. It has to be underlined that such effect was strain dependent; it was greater for the non-persistent strain. RTE meat products may be contaminated by L. monocytogenes and for this reason the potential of bacteriocins to control

241 it has been extensively investigated (33). In dry-cured ham the anti-listerial effect has been previously proven for enterocins $A B$ (13). In this previous study, enterocins $A B$ drastically reduced by $2.5 \log _{10} \mathrm{CFU} / \mathrm{g}$ L. monocytogenes in dry-cured ham stored at $4{ }^{\circ} \mathrm{C}$ for 1 day. The 244 results of our study confirm the potential of enterocins AB to impact on the viability of $L$. monocytogenes.

246 When the bacteriocinogenic E. faecalis was co-inoculated in the sliced dry-cured ham, the 247 evolution of the pathogen's population showed a reducing trend with time. The reduction 248 observed however cannot be considered important; in the case of strain S4-2 it was of 0.2 Log 249 between time zero and 7 days (statistically significant difference, $P<0.05$ ) while for strain 250 S12-1 it was of 0.1 . Therefore, the microbial competition exerted by E. faecalis resulted in 251 containment of L. monocytogenes, when compared to the control condition. It has to be underlined that the effective production of bacteriocin by E. faecalis in situ, after inoculation 253 in the dry-cured ham, was verified throughout the conservation period by AWDA (data not 254 shown). However, the results obtained with the enterocin and the E. faecalis cannot be 255 compared. Importantly, the E. faecalis strain used was not the same as the one from which the 256 enterocin was purified, but also it is clear that other variables such as bacteriocin liberation 257 from the cell and diffusion in the sliced ham most likely influence the effect of the E. faecalis 258 that was observed. The use of bacteriocinogenic cultures has been largely explored for 259 fermented foods, including fermented meat products. In the case of fermented meat products, 260 the bacteriocin producing strains used act as starter culture and contribute to the safety, by 261 microbial competition, bacteriocin and lactic acid production and to the development of the 
desired organoleptic properties of the final product (7). In non-fermented meat products, bacteriocin producing lactic acid bacteria may be added as protective cultures and they are

264 not expected to grow significantly or to produce large amounts of lactic acid. This approach 265 has not yet been explored for dry-cured ham and the results of the present study imply that 266 the E. faecalis strain used cannot by itself reduce the population of L. monocytogenes. This may be due to limited diffusion of the bacteriocin, or to its production at concentrations that may interfere with regulatory mechanisms and therefore contain growth, but not necessarily high enough to kill L. monocytogenes (4). Further the observed lack of lethal effect may be due to limited interaction of the two microorganisms in the solid food matrix, where physical contact, which has been proposed as an inter-species inhibitory mechanism (33), does not take place.

\section{Effect of enterocin and Enterococcus faecalis on Listeria monocytogenes gene expression} Although the effect of bacteriocins and bacteriocinogenic microorganisms on growth and inactivation behavior is widely investigated, the consequences on the physiology of the microorganisms have not been adequately addressed. The outcome of a given environmental condition on the physiological state can be inferred from the transcriptome, proteome or metabolome of microorganisms. Studies so far have primarily focused on the transcriptome under in vitro conditions $(9,25)$ to describe the impact of food-related environmental factors on the physiology and behavior of foodborne pathogens. The purpose of the present study was to explore the effect of an enterocin and a bacteriocin producing E. faecalis on expression of selected genes of L. monocytogenes, artificially inoculated in dry-cured ham.

284 Figures 1 and 2 present the relative gene expression for two different strains of $L$. monocytogenes; strain S4-2 (Figure 1) is a persistent strain while strain S12-1 (Figure 2) is a non-persistent strain. The genes chosen (Table 1) are representatives of stress response and 
virulence genes and have been previously employed in studies of L. monocytogenes gene expression in situ (19). Relative gene expression was calculated using as a control condition $L$. monocytogenes artificially inoculated in dry-cured ham. Therefore, figures 1 and 2 depict the 290 sole impact of enterocin or E. faecalis addition while the stressful conditions (low $\mathrm{a}_{\mathrm{w}}$, 291 refrigeration temperature, nitrites) that are known to have influence on gene expression, are 292 leveled out. During the long-term storage of vacuum packed dry cured ham, changes in the 293 physicochemical or microbiological parameters are not significant and therefore gene 294 expression is not expected to be influenced. Therefore, the gene expression was monitored up 295 to the $7^{\text {th }}$ day of refrigerated storage while a time point very close to the inoculation (30 296 minutes) was considered in order to capture the response of L. monocytogenes upon 297 inoculation. As can be seen in the two figures, the expression of the target genes fluctuated 298 during refrigerated conservation. Notably, for both strains of L. monocytogenes an overall 299 downregulation tendency for all the genes was observed after 30 minutes of storage. For 300 strain S12-1, this downregulation was already evident immediately after inoculation (time 0 ). 301 For strain S4-2, statistically significant variation in expression was observed for gene 302 Imo0669. This gene, encoding for a protein similar to an oxidoreductase and likely involved in 303 acid stress response, was downregulated at 30 minutes and then significantly upregulated at 3046 hours while expression leveled off throughout the rest of the storage period. Similar pattern 305 was observed for this gene in the strain S12-1; downregulation at 30 minutes, upregulation at 3066 hours followed in this case by significant up regulation at 168 hours. Upregulation at 168 307 hours was also observed for gene Imo2434, encoding for a glutamate decarboxylase and 308 involved in acid stress response. The virulence gene $\operatorname{prfA}$, encoding for a major virulence 309 transcriptional regulator, displayed fluctuating expression with a tendency for reduced 310 expression as compared to the condition of dry-cured ham. 
311 Apart from the effect of a bacteriocin extract we sought to investigate how the presence of a 312 bacteriocinogenic E. faecalis strain would influence gene expression of $L$. monocytogenes in 313 sliced dry-cured ham. The goal was to mimic a situation, i.e. co-presence in food of $L$. 314 monocytogenes and a competitive lactic acid bacterium, which is frequently verified during 315 food production and storage. For strain S4-2 no significant differences in gene expression were observed during time (data not shown). Contrarily, for strain S12-1 gene expression varied with time. As can be seen in figure 3, the main outcome observed from the presence of 318 E. faecalis in the dry-cured ham is downregulation for all genes throughout time with the exception of the 30 minutes time point in which all target genes were upregulated. Variation in gene expression through time resulted to be significant for genes Imo1421 and Imo0669. Limited information is available in the literature concerning the effect of bacteriocins or bacteriocin producing microorganisms on L. monocytogenes gene expression. Winkelströter and Martinis (32) registered dowregulation of the expression of inlA gene, an important virulence gene, in in vitro tests with 10 strains of L. monocytogenes, in the presence of 3 different bacteriocins, produced by E. faecium, Leuconostoc mesenteriodes and Lactobacillus sakei. Gene inlA, as well as prfA, encoding for a major virulence gene regulator, were downregulated in L. monocytogenes in the presence of metabolic products of two strains of $E$. faecium (34). The results of the present study are in agreement with these previous reports; gene prfA was downregulated in both L. monocytogenes strains, in response to the presence of the enterocin or the E. faecalis strain (for L. monocytogenes S12-1). Apart from prfA, also other genes (involved in virulence and stress response/adaptation) tested in the present study but also by Ye et al. (34) were downregulated in the presence of either a bacteriocin, a metabolic product of E. faecium or E. faecalis. Although this general trend was identified in both studies, it should be noted that the effect on gene expression depended both on the strain of $L$. 
a similar study, Miranda et al (20) investigated gene expression of L. monocytogenes in milk co-inoculated with a nisin-producing L. lactis. Out of the 4 genes tested, gadD2 consistently showed increased expression in the milk containing L. lactis compared to milk without $L$. lactis. Genes $\operatorname{sig} B$, groEL were also investigated and expression varied with time, showing a downregulation as incubation proceeded. On the other hand, gene $g b u$ was downregulated by the presence of L. lactis. Although the incubation temperature was different than in the current study (20 or $30^{\circ} \mathrm{C}$ as opposed to $7^{\circ} \mathrm{C}$ here), a liquid food matrix rather than a solid, and importantly, the antagonistic microorganism was different. Results concerning the $g b u$ gene appear to be consistent; in both studies the gene was essentially downregulated by the presence of a bacteriocin-producing microorganism.

When the two tested conditions, i.e. presence of enterocin and presence of E. faecalis, were compared (Figure 4), it was evident that the effect on gene expression was similar. With the exception of the 30 minutes time point when most genes were upregulated by the presence of E. faecalis, in the remaining time points expression went down. It is interesting to note that $E$. faecalis exhibited higher, mostly negative impact, on gene expression of L. monocytogenes compared to the enterocin. In most cases E. faecalis accentuated the downregulation of genes or inversed the pattern (from upregulated to downregulated). Expression of prfA was reduced in the presence of E. faecalis in 3 time points (at 6, 24 and 168 hours). Similarly, gene Imo0669 showed decreased expression in 4 out of 5 time points and this reduced expression was significant at 6 and 168 hours. Gene Imo1421 was further downregulated due to the presence of E. faecalis at the first time point.

Previous studies have addressed the effect of bacteriocins on gene expression of $L$. monocytogenes however data comparison is not plausible due to differences in the experimental approaches adopted. Different strains of L. monocytogenes tested, different media or types of food, different temperature/time regimes considered and a range of genes 
361 targeted. Concordant conclusions though have been reached and are also supported by the 362 present study. Bacteriocins or bacteriocin producing microorganisms have an effect on gene 363 expression of L. monocytogenes, both in vitro and in situ, and gene expression varies with time $364(20,15,34$, present study). These concordant outcomes suggest that $L$. monocytogenes senses 365 and responds by adapting its expression and therefore there is a need to go beyond viable 366 counts when biopreservation approaches are investigated and explore global physiological 367 response of the target microorganism.

368 369 
371 Viability of L. monocytogenes in dry-cured ham was greatly influenced by the addition of an 372 enterocin while the effect of the addition of E. faecalis was less pronounced. Differences were 373 detected between the two strains of L. monocytogenes; inhibition of the non-persistent strain 374 was more prominent compared to the persistent strain. The results obtained suggest that 375 addition of a bacteriocin is a more effective measure to control L. monocytogenes than 376 addition of a bacteriocinogenic protective culture, in sliced dry-cured ham. It remains to be 377 seen if the persistence phenotype is associated with higher resistance to a bacteriocin. 378 Further studies are needed to elucidate this aspect. On the other hand, a common pattern 379 regarding the expression of the 5 tested genes could be delineated for both strains; in the 380 presence of enterocin, the 30 minutes time point determined a downregulation of the genes 381 and this trend was essentially maintained throughout the storage period, up to 168 hours. For 382 the persistent strain, no significant differences could be observed in gene expression during 383 storage, in the presence of E. faecalis. On the contrary, for the non-persistent differences were 384 highlighted during storage, with an important shift between time 0 (downregulation), 30 385 minutes (upregulation) and the remaining period (downregulation). Based on the data of this 386 study we cannot correlate the persistence phenotype with the behavior observed; additional 387 strains (both persistent and non-persistent) should be tested, under in situ conditions, to 388 respond to this query. The gene expression results, although not conclusive, underline the 389 need to broaden our understanding of L. monocytogenes behavior in foods by integrating 390 phenotypic description with transcriptomic data.

392 Acknowledgements: This work has received financial support from International Committee 393 on Food Microbiology and Hygiene (ICFMH), project RTA2013-00070-C03-01 (Spanish 
Ministry of Economy and Competitiveness, MINECO) and the government of Chile (CONICYT scholarship).

\section{References}

398 1) Bae, D., C. Liu, T. Zhang, M. Jones, S.N. Peterson and C. Wang. 2012. Global gene expression of Listeria monocytogenes to salt stress. J. Food Prot. 75: 906-912.

2) Barefoot, S. F. and T. R. Klaenhammer. 1983. Detection and activity of lactacin B, a bacteriocin produced by Lactobacillus acidophilus. Appl. Environ. Microbiol. 45: 1808-1815.

3) Bover-Cid, S., N. Belletti, M. Garriga and T. Aymerich. 2011. Model for Listeria monocytogenes inactivation on dry-cured ham by high hydrostatic pressure processing. Food Microbiol. 28: 804-809.

5) Dal Bello, B., K. Rantsiou, A. Bellio, G. Zeppa, R. Ambrosoli, .T. Civera, T and L. Cocolin. 2010.

4) Chikindas, M., L., R. Weeks, D. Drider, V. A. Chistyakov and L. M. T. Dicks. 2018. Functions and emerging applications of bacteriocins. Curr Opin. Biotechnol. 49:23-28.

6) Ferrentino, G., S. Balzan and S. Spilimbergo. 2013. Supercritical carbon dioxide processing of dry cured ham spiked with Listeria monocytogenes: inactivation kinetics, color, and sensory evaluations. Food Bioprocess Technol. 6: 1164-1174.

7) Franciosa, I., V. Alessandria, P. Dolci, K. Rantsiou and L. Cocolin. 2018. Sausage fermentation and starter cultures in the era of molecular biology methods. Int. J. Food Microbiol. 279: 26-32

8) Garriga, M., T. Aymerich, S. Costa, J. M. Monfort and M. Hugas. 2002. Bactericidal synergism through bacteriocins and high pressure in a meat model system during storage. Food Microbiol. 19: 509-518. 
9) Greppi, A. and K. Rantsiou. 2016. Methodological advancements in foodborne pathogen determination: from presence to behavior. Curr. Opin. Food Sci. 8:80-88.

10) Holzapfel, W. H., R. Geisen and U. Schillinger. 1995. Biological preservation of foods with reference to protective cultures, bacteriocins and food-grade enzymes. Int. J. Food Microbiol. 24: $343-362$.

11) Hoz, L., M. I. Cambero, M. C. Cabeza, A. M. Herrero and J. A. Ordóñez. 2008. Elimination of Listeria monocytogenes from vacuum-packed dry-cured ham by E-beam radiation. J. Food Prot. 71: 2001-2006.

12) James, C., B. A. Onarinde and S. J. James. 2017. The Use and performance of household refrigerators: a review. Compr Rev Food Sci Food Saf. 16: 160- 179.

13) Jofré, A., T. Aymerich, J. M. Monfort and M. Garriga. 2008. Application of enterocins A and $\mathrm{B}$, sakacin $\mathrm{K}$ and nisin to extend the safe shelf-life of pressurized ready-to-eat meat products. Eur Food Res. Technol. 228: 159-162.

14) Kazmierczak, M.J., M. Wiedmann and K. J. Boor. 2006. Contributions of Listeria monocytogenes sigmaB and prfA to expression of virulence and stress response genesduring 448 extra- and intracellular growth. Microbiol. 152: 1827-1838.

16) Lindgren, S. E., and W. J. Dobrogosz. 1990. Antagonistic activities of lactic acid bacteria in food and feed fermentations. FEMS Microbiol. Rev. 87: 149-164.

17) Livak, K. J. and T. D. Schmittgen. 2001. Analysis of relative gene expression data using real- time quantitative PCR and the $2-\Delta \Delta C_{T}$ Method. Methods 25: 402-408.

18) Malekmohammadi, S., K. K. Kodjovi, J. Sherwood and T. M. Bergholz. 2017. Genetic and 
environmental factors influence Listeria monocytogenes nisin resistance. J. Appl. Microbiol. 123: $262-270$.

19) Mataragas, M., F. Rovetto, A. Bellio, V. Alessandria, K. Rantsiou, L. Decastelli and L. Cocolin. 2015. Differential gene expression profiling of Listeria monocytogenes in Cacciatore and Felino salami to reveal potential stress resi stance biomarkers. Food Microbiol. 46: 408-417.

20) Miranda, R, O., M. E. M. Campos-Galvão and L. A. Nero. 2017. Expression of genes associated with stress conditions by Listeria monocytogenes in interaction with nisin producer Lactococcus lactis. Food Res. Int. 105: 897-904.

21) Morales, P., J. Calzada and M. Nuñez. 2006. Effect of high-pressure treatment on the survival of Listeria monocytogenes Scott A in sliced vacuum-packaged Iberian and Serrano cured hams. J. Food Prot. 69: 2539-2543.

22) Pérez-Baltar, A., A. Serrano, D. Bravo, R. Montiel and M. Medina. 2019. Combined effect of high pressure processing with enterocins or thymol on the inactivation of Listeria monocytogenes and the characteristics of sliced dry-cured ham. Food Bioprocess Tech. 12: 288-297.

23) Ortiz, S., V. Lopez, D. Villatoro, P. Lopez,, J. C. Dávila and J. V. Martınez-Suarez. 2010. A 3year surveillance of the genetic diversity and persistence of Listeria monocytogenes in an Iberian pig slaughterhouse and processing plant. Foodborne Pathog. Dis. 7: 1177-1184. 
26) Rodríguez, E., B. González, P. Gaya, M. Nuñez and M. Medina. 2000. Diversity of bacteriocins produced by lactic acid bacteria isolated from raw milk. Int. Dairy J. 10: 7-15.

27) Schillinger, U., R. Geisenand and W. H. Holzapfel. 1996. Potential of antagonistic microorganisms and bacteriocins for the biological preservation of foods. Trends Food Sci. Technol. 71: 58-64.

28) Stiles M. E. 1996. Biopreservation by lactic acid bacteria. Antonie Van Leeuwenhoek. 70: 331-345.

29) Sue, D., K. J. Boor and M. Wiedmann. 2003. Sigma(B)-dependent expression patterns of 503 compatible solute transporter genes opиCA and Imo1421 and the conjugated bile salt hydrolase gene bsh in Listeria monocytogenes. Microbiol. 149: 3247- 3256.

30) Sue, D., D. Fink, M. Wiedmann and K. J. Boor. 2004. $\sigma^{\mathrm{B}}$-dependent gene induction and expression in Listeria monocytogenes during osmotic and acid stress conditions simulating the 509 intestinal environment. Microbiol. 150: 3843-3855.

33) Woraprayote, W., Y. Malila, S. Sorapukdee, A. Swetwiwathana, S. Benjakul and W. Visessanguan. 2016. Bacteriocins from lactic acid bacteria and their applications in meat and meat products. Meat Sci. 120: 118-132.

34) Ye, K., X. Zhang, Y. Huang, J. Liu, M. Liu and G. Zhou. 2018. Bacteriocinogenic Enterococcus faecium inhibits the virulence property of Listeria monocytogenes. LWT - Food Sci. Technol. 89: 87-92. 
527 35) Zilelidou, E. and P. N. Skandamis. 2018. Growth, detection and virulence of Listeria 528 monocytogenes in the presence of other microorganisms: microbial interactions from species 529 to strain level. Int. J. Food Microbiol. 277: 10-25.

530

531

532

533

534

535

536

537

538

539 


\section{Figure legends}

541 Figure 1. Relative gene expression for genes $\operatorname{prfA}, g b u B, \operatorname{lmo1421}, \operatorname{Imo2434}$, Imo0669 of Listeria monocytogenes strain S4-2 inoculated in dry-cured ham and supplemented with enterocin. Relative gene expression was calculated by the $2-\Delta \Delta \mathrm{C}_{\mathrm{T}}$ method and $\log 2$ values are reported. Error bars indicate standard deviation of two biological replicates. For gene 545 Imo0669, the asterisk indicates difference $(P<0.05)$ in the expression level, between 30 minutes and 6 hours of conservation.

547 Figure 2. Relative gene expression for genes $\operatorname{prfA}, g b u B, \operatorname{lmo1421}, \operatorname{Imo2434}$, Imo0669 of 548 Listeria monocytogenes strain S12-1 inoculated in dry-cured ham and supplemented with 549 enterocin. Relative gene expression was calculated by the $2-\Delta \Delta \mathrm{C}_{\mathrm{T}}$ method and $\log 2$ values are 550 reported. Error bars indicate standard deviation of two biological replicates. For genes $\operatorname{prfA}$, 551 Imo2434 and Imo0669, the asterisks indicate differences $(P<0.05)$ in the expression level, 552 across different time-points during conservation.

553 Figure 3. Relative gene expression for genes prfA, Imo1421, Imo2434, Imo0669 of Listeria 554 monocytogenes strain S12-1 co- inoculated in dry-cured ham with Enterococcus faecalis B1. 555 Relative gene expression was calculated by the $2-\Delta \Delta \mathrm{C}_{\mathrm{T}}$ method and $\log _{2}$ values are reported. 556 Error bars indicate standard deviation of two biological replicates. For genes Imo1421 and 557 Imo0669, the asterisks indicate differences $(P<0.05)$ in the expression level, across different 558 time-points during conservation.

559 Figure 4. Relative gene expression for genes prfA, Imo1421, Imo2434, Imo0669 of Listeria 560 monocytogenes strain S12-1 inoculated in dry cured ham supplemented with enterocin 561 (condition $a$ ) or co- inoculated in dry-cured ham with Enterococcus faecalis B1 (condition $b$ ). 562 Relative gene expression was calculated by the $2-\Delta \Delta \mathrm{C}_{\mathrm{T}}$ method and $\log _{2}$ values are reported. 563 Error bars indicate standard deviation of two biological replicates. Asterisks indicate 564 statistically significant differences (ANOVA, $p<0.05$ ) in the expression between conditions $a$ 565 and $b$. 